\title{
Preoperative biliary drainage by plastic or self-expandable metal stents in patients with periampullary tumors: results of a randomized clinical study
}

\section{(ㄷ)(i) $\Theta$}

\author{
Authors \\ Greger Olsson ${ }^{1,2}$, Farshad Frozanpor ${ }^{3}$, Lars Lundell ${ }^{1}$, Lars Enochsson ${ }^{1}$, Christoph Ansorge ${ }^{1}$, Marco Del Chiaro ${ }^{1}$, Marcus \\ Reuterwall-Hansson ${ }^{1}$, Alysha Shetye ${ }^{1}$, Urban Arnelo ${ }^{1}$
}

Institutions

1 Centre for Digestive Diseases, Karolinska University Hospital and Division of Surgery, CLINTEC, Karolinska Institutet, Stockholm, Sweden

2 Department of Surgery, Highland Hospital, Eksjö, Sweden

3 Department of Surgery, Danderyd Hospital, Stockholm, Sweden

submitted 15.12 .2016

accepted after revision 15.3.2017

\author{
Bibliography \\ DOI https://doi.org/10.1055/s-0043-110565 | \\ Endoscopy International Open 2017; 05: E798-E808 \\ (c) Georg Thieme Verlag KG Stuttgart · New York \\ ISSN 2364-3722
}

Corresponding author

Greger Olsson, MD, Department of Surgery, Highland

Hospital, SE-575 81 Eksjö, Sweden

greger.olsson@ki.se

\section{ABSTRACT}

Background and study aims Preoperative biliary drainage in patients with periampullary tumors and jaundice has been popularized to improve the quality of life and minimize the risks associated with subsequent radical surgery. The aim of this study was to investigate the possible superiority of self-expandable metal stents (SEMS) over plastic stents, by comparing the amount of bacteria in intraoperatively collected bile and using this variable as a proxy for the efficacy of the respective biliary drainage modalities.
Patients and methods In this randomized clinical trial, 92 patients with obstructive jaundice were enrolled; 45 were allocated to the plastic stent group and 47 to the SEMS group. The primary outcome was the extent and magnitude of biliary bacterial growth at the time of surgical exploration. Secondary outcomes were: macroscopic grading of inflammation of the stented bile ducts, occurrence of adverse events after stenting, stent dysfunction, recognized surgical complexities, and incidence of postoperative complications.

Results The patients were well matched regarding clinical and disease-specific characteristics. At surgery, there were no group differences in the bacterial amount and composition of the bile cultures or the perceived difficulty of surgical dissection. During the preoperative biliary drainage period, more instances of stent dysfunction requiring stent replacement were recorded in the plastic stent group (19\% vs. $0 \% ; P=0.03)$. Postoperative complications in patients who underwent curative surgery were more common in patients with plastic stents ( $72 \%$ vs. $52 \%$ ), among which clinically significant leakage from the pancreatic anastomoses seemed to predominate ( $12 \%$ vs. $3.7 \%$ ); however, none of these differences in postoperative adverse events reached statistical significance.

Conclusion This randomized clinical study was unable to demonstrate any superiority of SEMS in the efficacy of preoperative bile drainage, as assessed by the amount of bacteria in the intraoperatively collected bile. However, some data in favor of SEMS were observed among the clinical secondary outcomes variables (preoperative stent exchange rates) without increases in local inflammatory reactions.

Trial registered at ClinicalTrials.gov (NCT00501176).

\section{Introduction}

Patients with periampullary tumors frequently present with obstructive jaundice, which eventually leads to hepatic dysfunction, coagulopathy, biliary infections, malabsorption, and other digestive impairments [1-5]. Endoscopic biliary drainage is widely available and can be readily executed, and is therefore frequently utilized to relieve biliary obstruction, thereby optimizing the patient's physical status and quality of life prior to radical surgery. Whereas earlier retrospective as well as pro- 
spective series have reported a significant benefit of re-establishing the enterohepatic bile salt circulation in terms of reduced postoperative morbidity and mortality compared with untreated jaundiced patients [1-5], other series have conversely reported an increase in major surgical complications, and even mortality, in patients undergoing such drainage [6-16]. Although several clinical studies have reported that biliary drainage brings favorable outcome pathophysiologically, the clinical relevance of this finding is uncertain. The results of a recent multicenter randomized trial and a subsequent meta-analysis offer strong support for the view that preoperative biliary drainage is followed by an increased rate of major complications. Although many of these complications occurred postprocedurally (i. e. after stent insertion) rather than after the final operation, these authors concluded that preoperative biliary drainage in general should be avoided for resectable tumors $[6,7,9,16]$. Nonetheless, in spite of conflicting evidence, biliary drainage is still used for biliary decompression in selected patients with cholangitis or severe jaundice prior to radical surgery in many centers, especially for the increasing number of patients who are offered neoadjuvant therapy for pancreatic cancer [17]. In addition, the ongoing centralization of pancreatic surgery to high-volume centers may prolong the waiting time for definitive treatment, making biliary decompression inevitable, even in patients not eligible for neoadjuvant therapy.

It seems incontrovertible that bactobilia is associated with increased infectious complications after pancreatectomies, where measures have to be taken to prevent contamination of the wound and the abdominal cavity, and that the drainage techniques used might well contribute to the bacterial contamination rate of the biliary system [18]. In a large series from a high-volume center, it was claimed that patients with preoperative stents experienced significantly higher rates of wound infections compared with those undergoing surgery without prior stent placement (10\% vs. $4 \%$ ) [14]. Moreover, bacterial cultures of infected surgical wounds showed a strong correlation with the microorganisms found on bile cultures obtained at the time of surgery $[14,19]$. Bactobilia caused by stent dysfunction may result in a higher incidence of surgical infectious complications, but not necessarily in clinically more severe infections [14]. Nonetheless, cultivated amounts and strains of bacteria of the intrahepatic bile may well act as a marker for the level of impairment of the enterohepatic circulation of bacteria and accordingly function as a sensitive marker of the level of efficacy of bile drainage.

One important factor behind the divergent opinions about the efficacy and effect of preoperative biliary drainage is the type of stent being used. The improved patency of self-expandable metal stents (SEMSs) compared with plastic stents has been proven in large trials for the palliative treatment of patients with extrahepatic biliary obstruction [20-24]. Whether SEMSs are superior to plastic stents in the preoperative biliary drainage setting has to be explored carefully $[25,26]$. Moreover, a concern has been expressed about the local reaction in the hepatoduodenal ligament, induced by SEMS, and the associated surgical difficulties related to the precision of the dissection and withdrawal of the stent [27-29]. Finally, it has been claimed that covered SEMSs might increase the risk of cholecystitis and pancreatitis by blocking the cystic and the pancreatic duct orifices.

To address these different concerns, we performed a prospective randomized, double-blind study in patients suffering from jaundice who were undergoing preoperative biliary drainage, with either plastic stents or covered SEMSs. The aim of the study was to investigate the possible superiority of SEMSs over plastic stents, by comparing the amount of bacteria in intraoperatively collected bile and using this variable as a proxy for the efficacy of the respective biliary drainage modalities.

\section{Patients and methods}

The present study compared the insertion of a fully covered SEMS (Permalume silicone interior covered Platinol stent; Boston Scientific, Marlborough, Massachusetts, USA) with conventional plastic stents (polyethylene). The endoscopist decided which SEMS length to use, most commonly $6 \mathrm{~cm}(n=37)$, depending on the anatomical circumstances and the length of the stenosis. At full expansion, all SEMSs reached an inner diameter of $10 \mathrm{~mm}$, whereas the plastic stents were $10 \mathrm{Fr}$ and most often $7 \mathrm{~cm}$ in length $(\mathrm{n}=35)$.

The inclusion criteria were patients with a resectable periampullary tumor that was considered suitable for radical surgery and who presented with unrelieved extrahepatic, obstructive jaundice (bilirubin $>50 \mu \mathrm{mol} / \mathrm{L}$ ). The exclusion criteria were unsuccessful cannulation at endoscopic retrograde cholangiopancreatography (ERCP), patients who had already been treated with a stent, patients not fit for major surgery, or patients staged as unresectable during the pre-randomization work-up.

Patients were included after having signed an informed consent form before the ERCP investigation and were randomized after introduction of the guidewire into the bile duct. Randomization was achieved by use of sealed envelopes in blocks of 10 patients. The patient was unaware of the group affiliation, as was the physician in charge of the management of the patient post-procedurally. The patients remained in the group to which they were initially allocated, in an intention-to-treat type of analysis, even if they were switched to the other stent type (plastic or SEMS) in cases of stent dysfunction that required stent exchange.

The patients were thereafter scheduled for surgery within 6 weeks. All patients were evaluated during multidisciplinary team meetings that took place after the ERCP procedure. Three patients who at this stage were found to have borderline resectable tumors were offered, and underwent, neoadjuvant chemotherapy, which postponed the resection to a later date.

Before each ERCP, blood was collected for liver function analysis (aspartate transaminase, alanine transaminase, alkaline phosphatase, and bilirubin), white cell count, and C-reactive protein (CRP). Cholecystitis and cholangitis were defined according to Tokyo guidelines using clinical findings, laboratory values, and diagnostic imaging [30]. Stent dysfunction was defined as an increase in bilirubin $>50 \mu \mathrm{mol} / \mathrm{L}$ caused either by occlusion of the stent or by stent migration. In cases of stent replacement, the allocated stent type was reinserted if possible. 


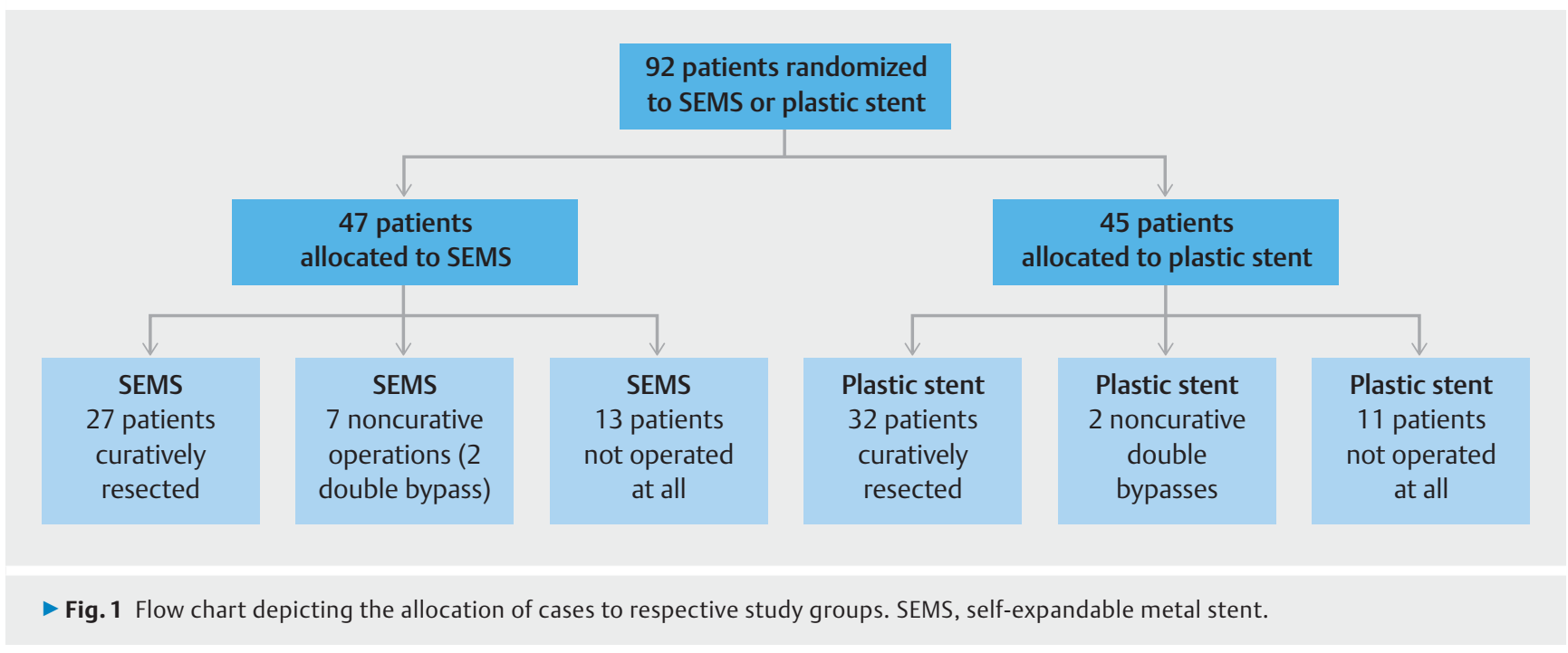

Pancreatitis was defined as abdominal pain in conjunction with elevated amylase at least three times the normal level at a time point more than 24 hours after the procedure [31].

During the initial part of the operation, the surgeon collected samples of bile from the common hepatic duct and from the gallbladder. The bile samples were transferred into culture media allowing for aerobic as well as anaerobic growth, the results of which were measured as colony-forming units per milliliter of bile (CFU/mL). During the procedure, the surgeon subjectively scored (easy, moderate, severe) the level of complexity of carrying out the lymph node dissection in the region of the hepatoduodenal ligament, construction of the hepaticojejunostomy, as well as the ease by which the stent could be withdrawn from the opened bile duct. The transection line of the common hepatic duct was examined through routine hematoxylin and eosin staining, after fixation in formalin, and the severity of inflammation was graded by the pathologist. A lymph node harvested from the hepatoduodenal ligament close to the bile duct transection line was also analyzed for the grade of inflammation (none, mild, moderate or severe). Postoperatively, the patients were transferred to a high dependency unit for dedicated postoperative care according to standard protocols.

A clinically significant pancreatic fistula was defined as a fistula requiring an adjustment in management (grade B) or any invasive therapeutic intervention (grade C) [32]. Delayed gastric emptying was classified largely according to the scoring system launched by the International Study Group on Pancreatic Surgery [33]. The postoperative complications were scored according to Clavien-Dindo [34]. Patients were followed up 4 6 weeks after surgery with an outpatient appointment.

The primary outcome measure of the study was to quantify the $\mathrm{CFU} / \mathrm{mL}$ in bile harvested during surgical exploration. Secondary outcomes were: stent dysfunction, preoperative macroscopic grading of inflammation of the bile ducts, the occurrence of adverse events after endoscopic stenting, the surgical difficulties in extracting the stent, the ease by which the hepatoduodenal dissection could be completed and the hepaticoje- junostomy could be constructed, and the incidence and severity of postoperative complications.

\section{Statistical analysis and ethics}

The required number of enrolled patients was estimated to be able to detect a $30 \%$ lower CFU/mL count in the SEMS group with a probability of $95 \%$ and a power of $80 \%$. In order to achieve this, a minimum of 60 matched patients needed to undergo surgical exploration and collection of bile samples. Thus, given the expected number of withdrawals resulting from findings that met the exclusion criteria during the subsequent preoperative work-up, at least 90 patients had to be randomized.

The data were analyzed using the statistical software JMP 9.0.0 (SAS Institute, Cary, North Carolina, USA). The demographic and clinical characteristics, endoscopic adverse events, intraoperative and postoperative findings, and complications are reported as medians and range or as number of cases and percentage of the total. The results of the bacterial cultures are reported as medians and interquartile range, as well as total sum of CFU/mL across all cases.

Categorical data were tested with Pearson's chi-squared test or Fisher's exact test when appropriate. When calculating the differences between means, the Student's $t$ test was used for data with a normal distribution. For numerical values not normally distributed, the Wilcoxon Rank-Sum Test was used. P values of $\leq 0.05$ were considered significant.

The study protocol was approved by the local ethics committee and written informed consent was received from all participating patients. All authors had access to the study data, and reviewed and approved the final manuscript. The trial was registered at ClinicalTrials.gov (NCT00501176).

\section{Results}

A total of 92 jaundiced patients were initially randomized into the trial, of whom 47 were allocated to receive an SEMS and 45 a plastic stent ( $\triangleright$ Fig. 1 ). During the course of the subsequent preoperative work-up, 13 SEMS and 11 plastic stent patients 
- Table 1 Demographic and clinical characteristics of patients undergoing exploratory surgery.

\begin{tabular}{|c|c|c|c|}
\hline & Plastic stent $n=34$ & SEMS $n=34$ & Pvalue \\
\hline \multicolumn{4}{|l|}{ Patient characteristics } \\
\hline BMI, median (range), $\mathrm{kg} / \mathrm{m}^{2}$ & $24.1(19.4-38.9)$ & $23.8(18.4-37.7)$ & 0.40 \\
\hline Age, median (range), years & $68(42-81)$ & $69(51-81)$ & 0.82 \\
\hline Sex, males, n (\%) & $23(67)$ & $17(50)$ & 0.14 \\
\hline Bilirubin, pre-ERCP, median (range), $\mu \mathrm{mol} / \mathrm{L}$ & $181(60-407)$ & $176(68-398)$ & 0.70 \\
\hline CRP, pre-ERCP, median (range), mg/L & $9(1-103)$ & $20(1-181)$ & 0.01 \\
\hline \multicolumn{4}{|l|}{ Tumor characteristics } \\
\hline Tumor size, median (range), mm & $25(10-44)$ & $25(5-60)$ & 0.89 \\
\hline Length of stenosis, median (range), mm & $15(5-70)$ & $20(5-30)$ & 0.28 \\
\hline Tumor stage (TNM), n (\%) & $\mathrm{n}=32$ & $n=33$ & \\
\hline - Stage I & $3(9)$ & $1(3)$ & 0.61 \\
\hline - Stage II & $19(59)$ & $25(76)$ & 0.13 \\
\hline - Stage III & $7(22)$ & $2(6)$ & 0.15 \\
\hline - Stage IV & $3(9)$ & $5(15)$ & 0.57 \\
\hline \multicolumn{4}{|l|}{ Tumor differentiation, n (\%) } \\
\hline - Well differentiated & $0(0)$ & $1(4)$ & 0.31 \\
\hline - Moderately differentiated & $15(50)$ & $10(40)$ & 0.21 \\
\hline - Poorly differentiated & $15(50)$ & $14(56)$ & 0.81 \\
\hline \multicolumn{4}{|l|}{ Tumor type, n (\%) } \\
\hline - Pancreatic & $27(79)$ & $29(85)$ & 0.52 \\
\hline - Biliary & $2(6)$ & $1(3)$ & 0.55 \\
\hline - Papillary & $4(12)$ & $3(9)$ & 0.69 \\
\hline - No tumor cells found & $1(3)$ & $0(0)$ & $>0.99$ \\
\hline - Duodenal & $0(0)$ & $1(3)$ & $>0.99$ \\
\hline Pancreatic tissue type, n (\%) & $\mathrm{n}=25$ & $\mathrm{n}=22$ & \\
\hline - Soft & $5(20)$ & $3(14)$ & 0.70 \\
\hline - Intermediate & $5(20)$ & $4(18)$ & $>0.99$ \\
\hline " Hard & $15(60)$ & $15(68)$ & 0.56 \\
\hline
\end{tabular}

were found to be nonresectable and did not undergo surgical exploration because of presence of either distant metastases or a locally advanced tumor. In addition, at surgery, which was performed a median of 6 weeks after randomization, seven SEMS patients and two plastic stent patients were found to be nonresectable because of distant metastases or the presence of a locally advanced tumor, leaving 27 and 32 patients, respectively, to undergo curative resection.

- Table 1 includes all patients who underwent surgery and shows that the two study groups were well matched regarding clinical and tumor-related characteristics. The CRP levels were significantly higher in the SEMS group, but the numerical differ- ence was clinically modest, only 20 vs. $9(P=0.01)$. There were more men in the plastic stent group (67\%) compared with the SEMS group (50\%), but the difference did not reach statistical significance $(P=0.14)$. - Table 1 also shows the risk profile of the pancreas (soft or hard) to develop subsequent leakage from the pancreaticojejunostomy; no differences in pancreatic hardness were seen between the two groups.

Details of bile cultures are shown in $>$ Table 2 , which contains only data from samples taken from the common hepatic duct, as similar results were observed in bile withdrawn from the gallbladder. Similar results were captured in SEMS and plastic stent patients with regard to both the total CFU/mL (131000 
- Table 2 Results of bacterial cultures from intraoperatively collected bile.

\begin{tabular}{|c|c|c|c|}
\hline & \multicolumn{3}{|l|}{ Bacterial cultures } \\
\hline & Plastic stent ( $n=27: 1$ bypass +26 resections) & SEMS ( $n=24$ resections) & $P$ value \\
\hline \multicolumn{4}{|c|}{ Bacteria count, median (IQR), CFU/mL } \\
\hline All bacteria species & $110000(100000-200000)$ & $131000(21050-210000)$ & 0.44 \\
\hline Gut bacteria & $100000(2000-160000)$ & $100000(11600-160000)$ & 0.58 \\
\hline \multicolumn{4}{|c|}{ Most commonly cultured bacteria species (total sum across each study group) } \\
\hline Plastic stent & $\mathrm{CFU} / \mathrm{mL}$ & SEMS & $\mathrm{CFU} / \mathrm{mL}$ \\
\hline Klebsiella spp. & 1450000 & Klebsiella spp. & 642000 \\
\hline Alpha Streptococcus spp. & 721000 & Enterococcus spp. & 581000 \\
\hline Enterococcus spp. & 510000 & Alpha Streptococcus spp. & 389000 \\
\hline Enterobacteriacae spp. & 543000 & Serratia spp. & 310000 \\
\hline Eschericia coli & 111000 & Enterobacteriacae spp. & 211000 \\
\hline Hemophilus spp. & 101000 & Eschericia coli & 205000 \\
\hline Hafnia spp. & 100000 & Hafnia spp. & 130000 \\
\hline Leclercia spp. & 100000 & Staphylococcus spp. & 110000 \\
\hline Serratia spp. & 80000 & Bacteroides spp. & 110000 \\
\hline \multirow[t]{7}{*}{ Proteus vulgaris } & 60000 & Streptococcus spp. & 100000 \\
\hline & & Aeromonas spp. & 100000 \\
\hline & & Candida spp. & 65000 \\
\hline & & Clostridium perfringens & 11000 \\
\hline & & Lactobacillus spp. & 10000 \\
\hline & & Streptotrophomonas spp. & 7000 \\
\hline & & Prevotella spp. & 5000 \\
\hline
\end{tabular}

vs. $110000 ; P=0.44)$ and the isolated species of bacteria with intestinal origin ( 100000 vs. $100000 ; P=0.58$ ).

During the preoperative work-up period, significantly more stent-related complications occurred in the plastic stent group ( $\triangleright$ Table 3), but otherwise no important differences between the study groups emerged. The stent dysfunction rates $(27 \%$ vs. $11 \% ; P=0.05)$ and stent exchange rates (24\% vs. $8 \% ; P=$ 0.05 ) in the group of all patients randomized differed significantly in favor of SEMS. The statistically significant difference remained in those patients undergoing curative surgery only regarding the stent exchange rate $(19 \%$ vs. $0 \%$; $P=0.03)$, but not in the stent dysfunction rate $(22 \%$ vs. $4 \%$; $P=0.06)$. On the other hand, the risk of post-ERCP pancreatitis was higher in the SEMS group ( $21 \%$ vs. $6 \% ; P=0.15$ ), but not significantly so.

The intraoperative observations, surgical data, and histological findings in the bile duct and adjacent lymph node are summarized in $>$ Table 4. More patients underwent explorative laparotomy without resection or bypass in the SEMS group (15\% vs. $0 \% ; P=0.05$ ). Otherwise there were no differences (either in all surgical patients or in only those who underwent curative surgery), in operational time, perioperative blood loss or technical difficulties in extracting the stent, creating the anastomosis or the grade of inflammation in the hepatoduodenal ligament, indicating that the different stent types did not affect the surgery technically.

Histopathologically, there was no difference in the degree of cholangitis in the ring specimen of bile duct harvested during surgery, but there was more sinus histiocytosis (foreign body reaction) in the lymph nodes in the hepatoduodenal ligament in the plastic stent group ( $26 \%$ vs. $7 \%$; $P=0.05)$. The postoperative course is summarized in $>$ Table 5 , both for the entire group undergoing exploratory surgery ( $>$ Table 5a) and for those patients who ultimately underwent curative resection ( $\triangleright$ Table 5b).

Patients undergoing curative surgery who had SEMSs had fewer complications ( $52 \%$ vs. $72 \% ; P=0.11$ ) and leakages from the anastomoses ( $4 \%$ vs. $12 \% ; P=0.36$ ) than patients with plastic stents. Similar differences were seen in the group of all surgical patients (overall complications $50 \%$ vs. $68 \%, P=0.14$; anastomotic leakage $3 \%$ vs. $12 \%, P=0.36$ ); however, infectious 
- Table 3 Preoperative adverse events and clinical characteristics at operation.

\begin{tabular}{|c|c|c|c|}
\hline & Plastic stent & SEMS & $P$ value \\
\hline All patients randomized, $n=92$ & $\mathrm{n}=45$ & $\mathrm{n}=47$ & \\
\hline \multicolumn{4}{|l|}{ Preoperative complications, n (\%) } \\
\hline - Stent dysfunction & $12(27)$ & $5(11)$ & 0.05 \\
\hline - Stent exchange & $11(24)$ & $4(9)$ & 0.05 \\
\hline - Cholecystitis & $1(2)$ & $4(9)$ & 0.36 \\
\hline - Cholangitis & $2(4)$ & $1(2)$ & 0.61 \\
\hline - Pancreatitis & $3(7)$ & $8(17)$ & 0.20 \\
\hline All patients undergoing surgery ${ }^{1}, n=68$ & $\mathrm{n}=34$ & $\mathrm{n}=34$ & \\
\hline \multicolumn{4}{|l|}{ Clinical characteristics at operation } \\
\hline - Bilirubin, median (range), $\mu \mathrm{mol} / \mathrm{L}$ & $22(2-363)$ & $18(4-166)$ & 0.13 \\
\hline - CRP, median (range), mg/L & $2(1-130)$ & $6(1-56)$ & 0.92 \\
\hline " Cholangitis preoperative, n (\%) & $6(18)$ & $2(6)$ & 0.26 \\
\hline - Prophylactic antibiotics at ERCP, n (\%) & $17(50)$ & $18(53)$ & 0.81 \\
\hline " Previous sphincterotomy, n (\%) & $3(9)$ & $1(3)$ & 0.61 \\
\hline - Precut sphincterotomy, n (\%) & $11(32)$ & $11(32)$ & $>0.99$ \\
\hline - Failed first cannulation at ERCP, n (\%) & $4(12)$ & $6(18)$ & 0.73 \\
\hline \multicolumn{4}{|l|}{ Preoperative complications, n (\%) } \\
\hline - Stent dysfunction & $7(21)$ & $2(6)$ & 0.15 \\
\hline - Pancreatitis & $2(6)$ & $7(21)$ & 0.15 \\
\hline - Cholecystitis & $1(3)$ & $3(9)$ & 0.61 \\
\hline - Cholangitis & $0(0)$ & $1(3)$ & $>0.99$ \\
\hline - Stent exchange & $6(18)$ & $1(3)$ & 0.11 \\
\hline All patients undergoing curative resection, $n=59$ & $\mathrm{n}=32$ & $\mathrm{n}=27$ & \\
\hline \multicolumn{4}{|l|}{ Clinical characteristics at operation } \\
\hline - Bilirubin at operation, median (range), $\mu \mathrm{mol} / \mathrm{L}$ & $21.5(2-363)$ & $18(4-166)$ & 0.16 \\
\hline - CRP, median (range), mg/L & $2.5(1-130)$ & $7.6(1-47)$ & 0.62 \\
\hline - Cholangitis preoperative, n (\%) & $6(19)$ & $2(7)$ & 0.27 \\
\hline - Prophylactic antibiotics at ERCP, n (\%) & $15(47)$ & $15(56)$ & 0.51 \\
\hline " Previous sphincterotomy, n (\%) & $3(9)$ & $0(0)$ & 0.24 \\
\hline - Precut sphincterotomy, n (\%) & $11(34)$ & $9(33)$ & 0.93 \\
\hline - Failed first cannulation at ERCP, n (\%) & $4(12)$ & $3(11)$ & $>0.99$ \\
\hline \multicolumn{4}{|l|}{ Preoperative complications, n (\%) } \\
\hline " Stent dysfunction & $7(22)$ & $1(4)$ & 0.06 \\
\hline - Pancreatitis & $2(6)$ & $3(11)$ & 0.65 \\
\hline - Cholecystitis & $1(3)$ & $2(7)$ & 0.59 \\
\hline - Cholangitis & $0(0)$ & $1(4)$ & 0.46 \\
\hline - Stent exchange, n (\%) & $6(19)$ & $0(0)$ & 0.03 \\
\hline
\end{tabular}


- Table 4 Intraoperative and histopathological findings in all patients undergoing surgery (curative and palliative).

\begin{tabular}{|c|c|c|c|}
\hline & Plastic stent $(n=34)$ & SEMS $(n=34)$ & Pvalue \\
\hline \multicolumn{4}{|l|}{ Operative data } \\
\hline Operation time, median (range), minutes & $398(165-565)$ & $395(134-627)$ & 0.66 \\
\hline Intraoperative bleeding, median (range), $\mathrm{mL}$ & $880(100-6800)$ & $800(100-6700)$ & 0.90 \\
\hline Vascular resection, n (\%) & $8(24)$ & $5(15)$ & 0.38 \\
\hline Total pancreatectomy, n (\%) & $2(6)$ & $3(10)$ & 0.65 \\
\hline Palliative double bypass, n (\%) & $2(6)$ & $2(6)$ & $>0.99$ \\
\hline Explorative laparotomy only, n (\%) & $0(0)$ & $5(15)$ & 0.05 \\
\hline \multicolumn{4}{|l|}{ Intraoperative findings, $\mathrm{n}$ (\%) } \\
\hline Subjective evaluation & $16(47)$ & $17(59)$ & 0.36 \\
\hline No inflammation & $18(53)$ & $12(41)$ & 0.65 \\
\hline Mild inflammation & $5(15)$ & $6(21)$ & 0.53 \\
\hline Moderate inflammation & $6(18)$ & $7(24)$ & 0.53 \\
\hline Severe Inflammation & $5(15)$ & $4(14)$ & $>0.99$ \\
\hline \multicolumn{4}{|l|}{ Difficulties creating hepaticojejunostomy } \\
\hline - None & $30(88)$ & $29(100)$ & 0.06 \\
\hline - Some & $3(9)$ & $0(0)$ & 0.24 \\
\hline - Severe & $1(3)$ & $0(0)$ & $>0.99$ \\
\hline Difficulties extracting stent & $0(0)$ & $0(0)$ & $>0.99$ \\
\hline Histopathological findings & $\mathrm{n}=34$ & $n=34$ & \\
\hline Histiocytosis & $9(26)$ & $2(7)$ & 0.05 \\
\hline - None & $25(74)$ & $27(93)$ & 0.04 \\
\hline - Mild & $5(15)$ & $1(3)$ & 0.20 \\
\hline - Moderate & $4(12)$ & $1(3)$ & 0.36 \\
\hline Cholangitis & $n=32$ & $n=25$ & \\
\hline - None & $1(3)$ & $0(0)$ & $>0.99$ \\
\hline - Mild & $11(34)$ & $8(32)$ & 0.85 \\
\hline - Moderate & $20(62)$ & $16(64)$ & 0.91 \\
\hline - Severe & $0(0)$ & $1(4.0)$ & 0.44 \\
\hline
\end{tabular}

complications were more common in the SEMS group (29\% vs. $15 \% ; P=0.14)$. None of these differences reached statistical significance.

\section{Discussion}

This study was designed to address the question of whether or not a covered SEMS can more effectively drain the obstructed bile duct system and restore the enterohepatic circulation of bacteria compared with plastic stents in jaundiced patients with periampullary tumors. The clinical need for preoperative biliary drainage in patients with potentially curable periampul- lary tumors remains controversial $[16,35]$. The most often cited study by van der Gaag et al. [16] demonstrated clearly that preoperative biliary drainage increased the morbidity. However, this study did not use SEMS, but rather plastic stents and sometimes even percutaneous transhepatic cholangiography drainage, which might induce more complications than modern SEMSs. However, adequate biliary drainage has clear pathophysiological benefits, and the mortality was significantly lower in the drained group in a recent meta-analysis [35]. In light of current developments in therapies for patients intended for curative resection, such as neoadjuvant chemotherapy treat- 
- Table 5a Postoperative adverse events in all patients undergoing surgery (curative and palliative).

\begin{tabular}{|c|c|c|c|}
\hline & Plastic stent $(n=34)$ & SEMS $(n=34)$ & Pvalue \\
\hline \multicolumn{4}{|l|}{ Perioperative outcomes } \\
\hline - Time from ERCP to surgery, median (range), days & $36(16-278)$ & $31(9-206)$ & 0.30 \\
\hline - Time in hospital, median (range), days & $16(4-46)$ & $14(7-65)$ & 0.94 \\
\hline - Time in HDU, mean (range), days & $3.4(0-9)$ & $3.6(0-10)$ & 0.84 \\
\hline \multicolumn{4}{|l|}{ Postoperative complications, n (\%) } \\
\hline " Overall & $23(68)$ & $17(50)$ & 0.14 \\
\hline - Surgical & $17(50)$ & $12(35)$ & 0.22 \\
\hline - Received antibiotics & $17(50)$ & $20(59)$ & 0.47 \\
\hline - Anastomotic leakage & $4(12)$ & $1(3)$ & 0.36 \\
\hline - Infection/abscess & $5(15)$ & $10(29)$ & 0.14 \\
\hline - Postoperative bleeding & $3(9)$ & $1(3)$ & 0.61 \\
\hline - Delayed gastric emptying & $8(24)$ & $7(21)$ & 0.77 \\
\hline - Reoperation & $2(6)$ & $1(3)$ & $>0.99$ \\
\hline - Wound dehiscence & $1(3)$ & $0(0)$ & $>0.99$ \\
\hline - Cardiopulmonary & $6(18)$ & $4(12)$ & 0.73 \\
\hline \multicolumn{4}{|l|}{ Complication severity, $\mathrm{n}(\%)$} \\
\hline - Clavien-Dindo I & $5(15)$ & $3(9)$ & 0.48 \\
\hline - Clavien-Dindo II & $10(29)$ & $6(18)$ & 0.25 \\
\hline - Clavien-Dindo III & $7(21)$ & $7(21)$ & $>0.99$ \\
\hline - Clavien-Dindo IV & $1(3)$ & $0(0)$ & $>0.99$ \\
\hline
\end{tabular}

ment, there remains a clinical incentive for patients with jaundice to undergo decompression [17].

We observed that the vast majority of intraoperative bile cultures contained bacteria in significant amounts. The rate of positive bile culture in our study was even higher than that found in most previous studies in which this issue has been adequately addressed $[13,18,36]$. The reasons behind this remain unclear, but we were unable to find a relationship between the actual length of the preoperative drainage or the type of stents used and the outcome of biliary cultures. It seems incontrovertible that bactobilia is associated with increased infectious complications after pancreatectomies and that the drainage technique contributes to a decreased bacterial contamination rate of the biliary system, which constituted one of the background prerequisites for the design and power calculation of the present study [18]. Based on current knowledge, it can be concluded that the profile and extent of bactobilia is not dependent on the type of drainage or the efficacy of the respective stent device, and does not co-vary with the clinical course during stent treatment. Similarly, based on the reasonable assumption that cultivated $\mathrm{CFU} / \mathrm{mL}$ of intrahepatic bile acts as a marker for the level of impairment of the enterohepatic circulation of bacteria, our study was unable to prove the superiority of SEMS in this specific regard. It is interesting to note that even in the per-protocol analysis, when we excluded those patients with plastic stents who required stent exchange and antibiotics because of stent dysfunction, we were still unable to detect a difference between the study groups. Antibiotic treatment is an important confounder as it has a prolonged effect on the amount and distribution of cultured bacteria in the biliary system $[13,14,18]$. Accordingly, the intention-to-treat analysis was exposed to the risk of diluting a possible effect of SEMS.

The length of drainage represents a pertinent issue. The advocates of preoperative drainage claim that the main reason why preoperative biliary drainage has not consistently been found to be beneficial was due to insufficient drainage time. Experimental observations suggest that liver function requires 4-6 weeks for recovery, even if the bilirubin level has returned to normal prior to 4 weeks. Therefore, the drainage time should be at least 4-6 weeks. In the present study, the median drainage time was 31 days (range $9-278$ days), although seven patients were offered neoadjuvant therapy initially and thus had prolonged drainage times; however only two of these patients eventually underwent curative resection. Together, all data 
- Table 5b Postoperative adverse events in patients undergoing curative resection.

\begin{tabular}{|c|c|c|c|}
\hline & Plastic stent $(n=32)$ & SEMS $(n=27)$ & $P$ value \\
\hline \multicolumn{4}{|l|}{ Perioperative outcomes } \\
\hline - Mortality, n (\%) & 0 & 0 & $>0.99$ \\
\hline - Time in hospital, median (range), days & $16(7-46)$ & $15(10-65)$ & 0.84 \\
\hline - Time in HDU, mean (range), days & $3.5(0-9)$ & $4.0(0-10)$ & 0.49 \\
\hline - Operation time, median (range), minutes & $402(229-565)$ & $420(290-627)$ & 0.42 \\
\hline - Intraoperative bleeding, median (range), mL & $1000(150-6800)$ & $1050(100-6700)$ & 0.87 \\
\hline - Vascular resection, n (\%) & $8(25)$ & $5(19)$ & 0.64 \\
\hline \multicolumn{4}{|l|}{ Postoperative complications, n (\%) } \\
\hline - Overall & $23(72)$ & $14(52)$ & 0.11 \\
\hline - Surgical & $17(53)$ & $12(44)$ & 0.51 \\
\hline - Received antibiotics & $17(53)$ & $15(56)$ & 0.85 \\
\hline - Anastomotic leakage & $4(12)$ & $1(4)$ & 0.36 \\
\hline - Infection/abscess & $5(16)$ & $6(22)$ & 0.51 \\
\hline - Postoperative bleeding & $3(9)$ & $1(4)$ & 0.62 \\
\hline - Delayed gastric emptying & $8(25)$ & $7(26)$ & 0.94 \\
\hline - Reoperation & $2(6)$ & $1(4)$ & $>0.99$ \\
\hline - Wound dehiscence & $1(3)$ & $0(0)$ & $>0.99$ \\
\hline - Cardiopulmonary & $6(19)$ & $4(15)$ & 0.74 \\
\hline \multicolumn{4}{|l|}{ Complication severity, n (\%) } \\
\hline - Clavien-Dindo I & $5(16)$ & $3(11)$ & 0.72 \\
\hline - Clavien-Dindo II & $10(31)$ & $4(15)$ & 0.22 \\
\hline - Clavien-Dindo III & $7(22)$ & $6(22)$ & 0.97 \\
\hline - Clavien-Dindo IV & $1(3)$ & $0(0)$ & $>0.99$ \\
\hline
\end{tabular}

suggest that a drainage time of $>4$ weeks can reduce overall morbidity compared with a shorter drainage time [14].

One of the controversies relating to preoperative biliary drainage is the idea that stent placement leads to local tissue inflammation and may make surgical resection more technically challenging, especially with SEMSs. We found that the type of stent did not affect the subjective assessment of surgical difficulty in dissecting the hepatoduodenal ligament, extracting the actual stent, or the complexity of completing the hepaticojejunostomy. Similarly, the histopathological results from the bile duct wall close to the proximal transection line, examined for the degree of inflammation, strengthened that conclusion. If anything, we observed more sinus histiocytosis (foreign body reaction) in the lymph nodes in the hepatoduodenal ligament in the plastic stent group. Even other objective measures such as bleeding and total operation time were not significantly different between the two groups. These results refute the suggested concern of an enhanced inflammatory reaction induced by the SEMS $[37,38]$.
Post-stent placement pancreatitis has been reported to occur more frequently in patients given fully covered SEMSs. This has been described as a function of the expansion of covered and uncovered metal stents causing compression of the pancreatic duct orifice [39]. However, many of these studies are small and of retrospective design, reporting on a nonconsecutive cohort of patients without comparison with a plastic stent. Furthermore, the type of SEMS used can influence the interpretation of the available study results. In the present study, numerically more patients with SEMSs developed pancreatitis compared with patients with a plastic stent $(21 \%$ vs. $6 \% ; P=$ $0.15)$, but this difference was not significant. This was also true for the development of cholecystitis, which was not found to be a significant clinical problem in either patient group.

A weakness of the study is that we chose bacterial counts in bile as the primary end point as opposed to relevant changes in complication rates. Accordingly, we were exposed to the risk of type II errors in the assessment of perioperative courses. Another weakness of the study is that a large proportion of the o- 
riginally randomized patients were excluded because they met exclusion criteria during the diagnostic work-up period, which also hampers the study by diluting the groups and thereby reducing the statistical power.

An issue to be addressed in the design of the study, is the possibility to completely blind for the allocation of stent type, although the endoscopist was not involved in the postoperative care. Special efforts have to be taken to maintain the blinding process, as the stent type can be disclosed by certain investigations carried out during the clinical management of the patients. In this regard, it should be mentioned that the majority of all tumor staging investigations were done before the insertion of the stent.

When considering overall complications, including noninfectious complications, we observed an increased need for reintervention in the plastic stent group, as represented by fewer stent failures $(P=0.06)$ and fewer instances of stent replacement in the SEMS group $(P=0.03)$. Although the results of the current randomized clinical study could not demonstrate any differences in bactobilia in perioperatively collected bile, it still offered arguments in favor of SEMS to optimize the preoperative course and the postoperative risk profile without the introduction of an enhanced risk for local complications. In previous studies it has been difficult to extract information about the true pros and cons of respective stent strategies, as only two studies, incorporating a limited number of patients, have compared plastic and metal stents for internal drainage [10, 40]. However, a recent Dutch multicenter trial, with a case-control study design, demonstrated a clear superiority of SEMS [15]. However, upfront surgery is still the preferred treatment strategy in patients presenting with jaundice due to periampullary tumors, and the pivotal trial-comparing preoperative biliary drainage using a fully covered SEMS versus upfront surgeryhas yet to be completed.

\section{Acknowledgment}

The authors thank Mr Luka Hrkac for his valuable contributions to data collection.

This study received grant support from County Council of Stockholm (SLL; ALF 20130512) and from Futurum, Jönköping County Council, Sweden.

\section{Competing interests}

None

\section{References}

[1] Bonin EA, Baron TH. Preoperative biliary stents in pancreatic cancer. J Hepatobiliary Pancreat Sci 2011; 18: 621-629

[2] Kloek JJ, Heger M, van der Gaag NA et al. Effect of preoperative biliary drainage on coagulation and fibrinolysis in severe obstructive cholestasis. J Clin Gastroenterol 2010; 44: 646-652
[3] Sewnath ME, Birjmohun RS, Rauws EA et al. The effect of preoperative biliary drainage on postoperative complications after pancreaticoduodenectomy. J Am Coll Surg 2001; 192: 726 - 734

[4] Smith RA, Dajani K, Dodd S et al. Preoperative resolution of jaundice following biliary stenting predicts more favourable early survival in resected pancreatic ductal adenocarcinoma. Ann Surg Oncol 2008; 15: $3138-3146$

[5] Velanovich V, Kheibek T, Khan M. Relationship of postoperative complications from preoperative biliary stents after pancreaticoduodenectomy. A new cohort analysis and meta-analysis of modern studies. JOP 2009; 10: $24-29$

[6] Fang Y, Gurusamy KS, Wang Q et al. Pre-operative biliary drainage for obstructive jaundice. Cochrane Database Syst Rev 2012; 9: CD005444

[7] Fang Y, Gurusamy KS, Wang Q et al. Meta-analysis of randomized clinical trials on safety and efficacy of biliary drainage before surgery for obstructive jaundice. Br J Surg 2013; 100: 1589-1596

[8] Hatfield AR, Tobias R, Terblanche J et al. Preoperative external biliary drainage in obstructive jaundice. A prospective controlled clinical trial. Lancet 1982; 2: 896-899

[9] Lai EC, Lau SH, Lau WY. The current status of preoperative biliary drainage for patients who receive pancreaticoduodenectomy for periampullary carcinoma: a comprehensive review. Surgeon 2014; 12: $290-296$

[10] Mezhir JJ, Brennan MF, Baser RE et al. A matched case-control study of preoperative biliary drainage in patients with pancreatic adenocarcinoma: routine drainage is not justified. J Gastrointest Surg 2009; 13 : $2163-2169$

[11] Povoski SP, Karpeh MSJr, Conlon KC et al. Association of preoperative biliary drainage with postoperative outcome following pancreaticoduodenectomy. Ann Surg 1999; 230: 131-142

[12] Sewnath ME, Karsten TM, Prins MH et al. A meta-analysis on the efficacy of preoperative biliary drainage for tumors causing obstructive jaundice. Ann Surg 2002; 236: 17-27

[13] Sivaraj SM, Vimalraj V, Saravanaboopathy P et al. Is bactibilia a predictor of poor outcome of pancreaticoduodenectomy? Hepatobiliary Pancreatic Dis Int 2010; 9: 65-68

[14] Sohn TA, Yeo CJ, Cameron JL et al. Do preoperative biliary stents increase postpancreaticoduodenectomy complications? J Gastrointest Surg 2000; 4: 258-567

[15] Tol JA, van Hooft JE, Timmer R et al. Metal or plastic stents for preoperative biliary drainage in resectable pancreatic cancer. Gut 2016; 65: $1981-1987$

[16] van der Gaag NA, Rauws EA, van Eijck CH et al. Preoperative biliary drainage for cancer of the head of the pancreas. N Engl J Med 2010; 362: $129-137$

[17] Aadam AA, Evans DB, Khan A et al. Efficacy and safety of self-expandable metal stents for biliary decompression in patients receiving neoadjuvant therapy for pancreatic cancer: a prospective study. Gastrointest Endosc 2012; 76: $67-75$

[18] Jagannath P, Dhir V, Shrikhande S et al. Effect of preoperative biliary stenting on immediate outcome after pancreaticoduodenectomy. $\mathrm{Br}$ ] Surg 2005; 92: 356-361

[19] Sahora K, Morales-Oyarvide V, Ferrone C et al. Preoperative biliary drainage does not increase major complications in pancreaticoduodenectomy: a large single center experience from the Massachusetts General Hospital. J Hepatobiliary Pancreat Sci 2016; 23: 181 - 187

[20] Davids PH, Groen AK, Rauws EA et al. Randomised trial of self-expanding metal stents versus polyethylene stents for distal malignant biliary obstruction. Lancet 1992; 340: 1488-1492

[21] Kaassis M, Boyer ], Dumas R et al. Plastic or metal stents for malignant stricture of the common bile duct? Results of a randomized prospective study Gastrointest Endosc 2003; 57: 178 - 182 
[22] Moses PL, Alnaamani KM, Barkun AN et al. Randomized trial in malignant biliary obstruction: plastic vs partially covered metal stents. World J Gastroenterol 2013; 19: 8638-8646

[23] Prat F, Chapat O, Ducot B et al. A randomized trial of endoscopic drainage methods for inoperable malignant strictures of the common bile duct. Gastrointest Endosc 1998; 47: 1-7

[24] Soderlund C, Linder S. Covered metal versus plastic stents for malignant common bile duct stenosis: a prospective, randomized, controlled trial. Gastrointest Endosc 2006; 63: 986 - 995

[25] Ahmad J, Siqueira E, Martin J et al. Effectiveness of the Ultraflex Diamond stent for the palliation of malignant biliary obstruction. Endoscopy 2002; 34: $793-796$

[26] Ferlitsch A, Oesterreicher C, Dumonceau JM et al. Diamond stents for palliation of malignant bile duct obstruction: a prospective multicenter evaluation. Endoscopy 2001; 33: 645-650

[27] Cavell LK, Allen PJ, Vinoya C et al. Biliary self-expandable metal stents do not adversely affect pancreaticoduodenectomy. Am J Gastroenterol 2013; 108: $1168-1173$

[28] Lawrence C, Howell DA, Conklin DE et al. Delayed pancreaticoduodenectomy for cancer patients with prior ERCP-placed, nonforeshortening, self-expanding metal stents: a positive outcome. Gastrointest Endosc 2006; 63: $804-807$

[29] Wasan SM, Ross WA, Staerkel GA et al. Use of expandable metallic biliary stents in resectable pancreatic cancer. Am J Gastroenterol 2005; 100: $2056-2061$

[30] Takada T, Strasberg SM, Solomkin JS et al. TG13: Updated Tokyo guidelines for the management of acute cholangitis and cholecystitis. J Hepatobiliary Pancreat Sci 2013; 20: 1 - 7
[31] Cotton PB, Lehman G, Vennes J et al. Endoscopic sphincterotomy complications and their management: an attempt at consensus. Gastrointest Endosc 1991; 37: $383-393$

[32] Bassi C, Dervenis C, Butturini G et al. Postoperative pancreatic fistula: an international study group (ISGPF) definition. Surgery 2005; 138: $8-13$

[33] Wente MN, Bassi C, Dervenis C et al. Delayed gastric emptying (DGE) after pancreatic surgery: a suggested definition by the International Study Group of Pancreatic Surgery (ISGPS). Surgery 2007; 142: 761 768

[34] Dindo D, Demartines N, Clavien PA. Classification of surgical complications: a new proposal with evaluation in a cohort of 6336 patients and results of a survey. Ann Surg 2004; 240: 205-213

[35] Sun C, Yan G, Li Z et al. A meta-analysis of the effect of preoperative biliary stenting on patients with obstructive jaundice. Medicine 2014; 93: e189

[36] Lermite E, Pessaux P, Teyssedou C et al. Effect of preoperative endoscopic biliary drainage on infectious morbidity after pancreatoduodenectomy: a case-control study. Am J Surg 2008; 195: 442-446

[37] Kahaleh M, Tokar J, Le T et al. Removal of self-expandable metallic Wallstents. Gastrointest Endosc 2004; 60: 640-644

[38] Petersen BT. SEMS removal: salvage technique or new paradigms. Gastrointest Endosc 2005; 62: $911-913$

[39] Cote GA, Kumar N, Ansstas M et al. Risk of post-ERCP pancreatitis with placement of self-expandable metallic stents. Gastrointest Endosc 2010; $72: 748-754$

[40] Mullen JT, Lee JH, Gomez HF et al. Pancreaticoduodenectomy after placement of endobiliary metal stents. J Gastrointest Surg 2005; 9: $1094-1104$ 